\title{
Professores sabem lidar com o suicídio em sala de aula? Limites e possibilidades na formação docente
}

Can teachers deal with suicide in a classroom? limits and possibilities in teaching training

DOI: $10.5935 / 2447-8539.20190008$

Valquíria de Souza Andrade

e-mail:valpolito@bol.com.br

\section{Resumo}

Este artigo analisa o atual cenário da formação de professores no Brasil e os limites e possibilidades que tais docentes possuem na medida em que tem acesso as informações sobre o tema suicídio entre os adolescentes e de que forma podem atuar no seu combate. 0 tema e de extrema importância, haja visa o aumento significativo de casos de suicídio e tentativa de suicídio entre adolescentes. Realizado uma busca bibliográfica no site da Biblioteca Virtual em Saúde (BIREME), com os seguintes descritores "Suicídio", "Adolescentes" e "Ensino", em que foram encontrados 417 artigos, porém foram utilizados somente artigos em português, com texto completo e que atendesse os objetivos do trabalho, sendo assim, foram usados 17 artigos. Conclui-se que a escola é um espaço privilegiado para a promoção e proteção da saúde, onde os adolescentes passam boa parte do tempo, porém a formação de professores voltado para o tema suicídio ainda é muito deficitária, carecendo de formação específica através de curso de capacitação e educação continuada.

Palavras-chave: Adolescentes. Ensino. Suicídio.

\section{Abstract}

This work aims to analyze the current scenario of education in Brazil and the limits and possibilities that teachers have in that they have to access the information about suicide among adolescents and how they can act to tackle it. The theme is of extreme importance, considering the significant increase of cases of suicide and attempted suicide among teenagers. A bibliographic search was performed on the Virtual Health Library website (BIREME) with the following descriptors: "Suicide", "Adolescents" and "Teaching", where 417 articles were found. But only17 full text articles in Portuguese were used, especially those that met the goals of this work. It was concluded that the school is a privileged space for the promotion and protection of health, where the adolescents spend a good part of their time. However, the training for teachers regarding the issue of suicide is still very deficient, lacking of specific training through a training and continuing education course.

Keywords: $\quad$ Adolescent. Teaching. Suicide. 


\section{INTRODUÇÃO}

O suicídio constitui-se num importante problema de saúde pública. Está entre as dez principais causas de morte na população mundial em todas as faixas etárias, ocupando o terceiro lugar no grupo com idade entre 15 e 34 anos (BAGGIO et al., 2009).

Numa perspectiva histórica, a adolescência é uma categoria sociocultural, percebível por meio de interpretações e diversos critérios, a depender da cultura na qual o indivíduo a vivência. É entendida também como parte do processo transitório do desenvolvimento humano entre a infância e a idade adulta, considerando-se as dimensões biopsicológica e social (FERREIRA et al., 2013).

Atualmente, a adolescência é considerada uma fase

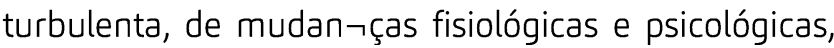
que envolve questionamentos afetivos, familiares e sociais. Além disso, necessidades afetivas e conflitos podem tornar o adolescente vulnerável às influências de seus pares e do ambiente sociocultural em que vive. Nessa fase da vida, torna-se necessário ao indivíduo o desenvolvimento de sua auto cognição, que implica a apreensão da individualidade biológica, psicológica e social (FERREIRA et al., 2013).

Além disso, entre adolescentes, os comportamentos de risco, em interação com fatores sociais e ambientais, têm gerado um aumento de mortes prematuras (BAGGIO et al., 2009).

Mas também, não podemos esquecer as condutas de risco à saúde (tabagismo, consumo de bebidas alcoólicas e uso de drogas) que faz com que o estresse psicossocial em adolescentes estudantes do ensino médio chegue a níveis alarmantes interferindo no aprendizado deles e ao mesmo tempo interferindo no andamento das atividades das escolas, comprometendo todo o ensino (CARVALHO et al., 2011).

$\mathrm{Na}$ literatura específica, com frequência o comportamento suicida é classificado em três categorias: ideação suicida, tentativa de suicídio e suicídio consumado (ARAUJO et al., 2010).

Entretanto, sabe-se que as estatísticas sobre os atos suicidas são falhas e subestimadas, uma vez que o número que consta nas estatísticas oficiais é extraído das causas de morte assinaladas nos atestados de óbito (ARAUJO et al., 2010).

Porém, estes nem sempre são confiáveis, pois tanto a família quanto a própria sociedade, comumente, pressionam para que a causa seja falsificada. Certamente a subestimação estatística será mais intensa quando se trata de crianças e adolescentes, em que os atos autodestrutivos serão negados ou até escondidos pela família, diante de maiores sentimentos de culpa e/ou vergonha pelo ato (ARAUJO et al., 2010).
Suicídio é uma palavra originada no latim, derivada da junção das expressões sui (si mesmo) e caederes (ação de matar). Num sentido geral, significa o ato voluntário por meio do qual o indivíduo possui a intenção e provoca a própria morte. Apesar de não existir uma definição única aceitável, sabe-se que implica necessariamente um desejo consciente de morrer e a noção clara do que 0 ato executado pode resultar (VIEIRA, 2008).

Conforme Barros, Coutinho Araújo \& Castanha (2006), tem-se observado, nas últimas décadas, que o comportamento suicida tem aumentado entre os jovens, sendo a adolescência uma fase bastante associada à morte por causas violentas.

É um período do desenvolvimento marcado por diversas modificações biológicas, psicológicas e sociais; e essas mudanças, geralmente, são acompanhadas de conflitos e angústias. Às vezes, quando expostos às intensas e prolongadas situações de sofrimento, de desorganização, os adolescentes podem desenvolver patologias e tornar-se mais vulneráveis ao suicídio (WERLANG et al., 2002).

É, também, nesse período que tanto as ideações quanto às tentativas ganham uma maior proporção, quando associadas ao quadro depressivo, embora essa situação não se restrinja somente à adolescência. Igualmente nesse período há uma melhor compreensão a respeito da morte pelo desenvolvimento do pensamento abstrato; portanto, é importante que se verifique a presença da ideação suicida, já que esta pode ser considerada como um antecedente a uma a possível culminância do ato suicida (SILVA et al., 2006).

Assim diante de um jovem que pensa, faz ameaças, tenta ou consuma o ato suicida, observa-se um colapso nos seus mecanismos adaptativos, os quais visam aliviar o sofrimento (BORGES, 2002).

Por isso, o trabalho foi desenvolvido no sentido de aprofundar nosso conhecimento sobre o tema suicídio entre os adolescentes e até que ponto os professores estão preparados para detectar de forma precoce uma possível vontade de um adolescente se suicidar e de que maneira o sistema educacional pode contribuir na saúde dos nossos adolescentes.

\section{METODOLOGIA}

Para a realização desta pesquisa, foi adotado o sistema de revisão de literatura, a qual se constitui na busca de informações acerca de determinado tema, com a finalidade de sistematizar a produção do conhecimento sobre um problema de pesquisa (LEITE et al, 2016).

Ao proporcionar os resultados das investigações, essa metodologia pode auxiliar profissionais, pesquisadores e estudantes em suas decisões, após conhecerem o que 
tem sido estudado sobre o assunto (MACHADO et al, 2016).

A busca bibliográfica foi desenvolvida na Biblioteca Virtual de Saúde (BVS-BIREME), refinando pelas fontes de dados Literatura Latino-Americana e do Caribe em Ciências da Saúde (LILACS) e Literatura Internacional em Ciências da Saúde (MEDLINE), no primeiro trimestre de 2019. Nessas bases de dados, foram realizadas consultas com os termos "Suicídio", "Adolescentes" e "Ensino".

Para a seleção dos estudos, adotaram-se os seguintes critérios de elegibilidade: artigos completos referente a pesquisas originais e revisões de literatura disponíveis no meio eletrônico pesquisado, publicados apenas em português que possuíam aderência ao objetivo proposto e que abordassem a temática estudada. Os critérios de exclusão foram: resumos, teses e dissertações, artigos não encontrados na íntegra, artigos em outros idiomas diferentes do estudado, também foram descartados e que não atendessem ao objetivo proposto por esta pesquisa.

A partir da leitura prévia dos títulos e resumos das produções encontradas, foram selecionados os artigos que se enquadraram nos critérios de inclusão.

Para o mapeamento das produções científicas, utilizouse uma ficha resumo constituída das variáveis, a fim de responder à questão norteadora, que contemplou os seguintes aspectos considerados: título do artigo; ano de publicação e periódicos; principais achados; publicação, objetivo e assunto principal, metodologia/tipo de estudo/descritores utilizados no artigo e idioma do artigo (LEITE et al, 2016).

Foi desenvolvida a análise de conteúdo, que conta com três etapas: pré-análise, exploração do material e interpretação dos resultados. Realizou-se leitura flutuante, a qual possibilitou ter uma visão abrangente do conteúdo. Enfim, a metodologia possibilitará conhecer os artigos que citam os aspectos envolvendo o suicídio dentro da sala de aula e de que forma o professor poderá atuar na prevenção deles.

\section{REVISÃO DE LITERATURA}

No Brasil, as pesquisas sobre comportamentos de saúde entre jovens ainda são escassas e se concentram em questões ligadas à gravidez precoce, ao uso de anticoncepcionais e ao uso de substâncias. Pouco se conhece sobre outros comportamentos na área de saúde e ainda menos quanto à sobreposição de diferentes comportamentos de risco entre jovens e adolescentes (GOUVEIA, et al., 2000).

Além do mais, a escola tem papel estratégico para a promoção e proteção da saúde dos alunos, pois é o local onde são reproduzidos os padrões de comportamentos e relacionamentos que podem pôr em risco a saúde dos jovens. Nesse sentido, acredita- se que se a escola possa ser um local privilegiado para a identificação precoce de situações problemáticas, já que aspectos relacionados ao meio familiar, grupo de amigos e escola são de extrema importância para a qualidade de vida do adolescente (BAGGIO et al., 2009).

Além disso, os estudos vêm mostrando que relações familiares adversas, pequeno número de amigos, contatos agressivos com colegas e sintomas depressivos aumentam a prevalência de planejamento suicida, esses sinais são muitas das vezes perceptíveis, por isso a necessidade do professor próximo desse aluno estar capacitado na detecção precoce da vontade em suicidar e com isso encaminhar ao serviço especializado mais próximo (BAGGIO et al., 2009).

O suicídio passou a ser encarado como um dos principais problemas de saúde em populações jovens, por isso a necessidade da participação da escola no seu combate. A exposição ao tabagismo, ao consumo de bebidas alcoólicas e ao uso de drogas na adolescência pode ser fator associado ao estresse psicossocial, e a análise desta inter-relação poderá oferecer subsídios ao planejamento de intervenções e de outras investigações (CARVALHO et al., 2011).

É importante também porque grande proporção de adolescentes refere estresse psicossocial, tais como sentimento de solidão, dificuldade para dormir devido à preocupação, sentimento de tristeza, pensamento de suicídio e planos de suicídio, também, o estresse psicossocial está associado também ao baixo desempenho escolar e envolvimento em atividades violentas e criminosas (CARVALHO et al., 2011).

Adolescentes com manifestações suicidas apresentam história positiva para situações de violência, brigas e agressões, transgressão às leis e problemas de conduta em gera (BAGGIO et al., 2009).

Com isso, a violência materializa-se em diversos espaços sociais e tem sido mais frequente, nos últimos anos, no ambiente escolar. Tal fato contradiz a concepção de escola como um espaço de socialização do conhecimento, formação e, sobretudo, de proteção (CASTRO et al., 2011).

No Brasil, a violência escolar tem sido objeto de investigações científicas. De depredações, invasões dos espaços escolares e brigas entre os grupos, a violência mudou para agressões de alunos contra professores. Observa-se comportamento de violência manifestado nas agressões com o uso de armas brancas, de fogo, consumo de drogas, preconceito, bullying, e atualmente cenário de suicídio entre os adolescentes (CASTRO et al., 2011).

O suicídio quando ocorre principalmente dentro da escola, também acomete além da população jovem outras pessoas, como por exemplo, professores, colegas 
de turma e amigos. O suicídio é concebido como violência auto infligida e componente de "causas externas" e tem aumentado na população jovem das cidades brasileiras, provocando impacto na saúde pública, por isso a importância dessa discussão no ambiente escolar (CASTRO et al., 2011).

Segundo Bahls (2002), em adolescentes há atualmente a compreensão de que a depressão maior é comum, debilitante e recorrente, envolvendo um alto grau de morbidade e mortalidade, especialmente através do suicídio, constituindo-se em uma das principais preocupações da saúde pública e, a maioria dos casos, não é identificada nem encaminhada para tratamento.

As pesquisas ressaltam que se deve estar atento para os seguintes sinais: os estados de humor irritável ou depressivo duradouro e/ou excessivo, os períodos prolongados de isolamento ou hostilidade com família e amigos; o afastamento da escola ou queda importante no rendimento escolar; o afastamento de atividades grupais e comportamentos como abuso de substâncias (álcool e drogas), violência física, atividade sexual imprudente e fugas de casa.

Enfim, a escola precisa se preocupar também com a vulnerabilidade. Há que se destacar que, além do componente social, deve saber sobre: vulnerabilidade individual e programática. Enquanto a vulnerabilidade social diz respeito ao acesso às informações, uso das informações e possibilidade de incorporação em mudanças práticas na vida cotidiana (sendo associada diretamente a recursos materiais e instituições sociais como a escola), a vulnerabilidade individual inclui aspectos cognitivos, sob a forma da disposição de informações aos indivíduos e capacidade de elaboração de tais dados, além de aspectos comportamentais, por meio de competência, interesse e desenvoltura, para modificar preocupações com atitudes e ações protegidas e protetoras (FERREIRA et al., 2013).

A vulnerabilidade programática, por seu turno, remetenos à junção dos componentes social e individual, correspondendo, de maneira geral, ao uso de indicadores - dentre outros recursos - para a avaliação de realidades. Assim, ressalta-se que a compreensão das vulnerabilidades às quais os adolescentes estão expostos pode ser captada a partir do entendimento da interligação dos componentes supracitados. 0 conhecimento desse padrão é primordial no estudo das vulnerabilidades do adolescente, pois permitem pela escola o desenvolvimento de estratégias preventivas (FERREIRA et al., 2013).

Destaca-se que o comportamento suicida ocorre, muitas vezes, como reflexo de conflitos internos, sentimentos de depressão e ansiedade que acompanham a profunda reorganização física, psíquica e social que ocorre na adolescência (BAGGIO et al., 2009).
Entretanto, pode ser difícil determinar a intencionalidade de atitudes autodestrutivas, por isso a necessidade de o professor em sala de aula estar sempre atendo aos sinais para prevenir.

Em função disso, estudos são feitos para mapear um conjunto de fatores de risco que, individualmente ou associados, contribuem para a ação, tais como: uso de álcool e drogas, dificuldades nas relações familiares, transtornos alimentares, baixa autoestima, exposição à violência, sentimentos depressivos, dentre outros. Assim, esses temas precisam estar inteiramente inseridos nos cursos de formação de professores, nos cursos específicos de graduação e pós-graduação destinados à formação docente e nos cursos de formação continuada e educação permanente, para que o docente esteja mais preparado para o trabalho em sala de aula (BAGGIO et al., 2009).

Além disso, as pesquisas apontam que a ideação suicida e a sua idade têm um importante valor preditivo para o ato suicida, por isso os cursos de formação de professores não podem negar essas características e de alguma forma precisa ser debatidos no meu do sistema educacional (ARAUJO et al, 2010).

Os adolescentes que apresentaram ideação se auto representam como pessoas sozinhas, associando a ideação a sentimentos de desesperança e solidão, ao mesmo tempo em que expressaram um pedido de ajuda diante de seu sofrimento. Os achados na presente revisão enfatizam a importância dos fatores sinalizadores, que podem permitir uma melhor compreensão sobre a problemática do suicídio (ARAUJO et al, 2010).

Alguns estudos apontam a importância do pensamento e comportamento suicida, os quais os professores precisam ser capacitados para detectar de forma precoce, haja vista que, além dos fatores externos já citados como o uso do álcool e violência, não pode esquecer-nos dos fatores internos de cada adolescente, como por exemplo a insatisfação corporal, obesidade, magreza, deficiência física e até mesmo alguns casos bullying na sala de aula que reflete na vida dos adolescentes dentro e fora da escola. Tais pesquisas aponta que os adolescentes em idade escolar, a escola pode ser um agente de discussão sobre a temática, auxiliando na prevenção da insatisfação corporal e desfechos suicidas. Outros profissionais envolvidos com essa população e os familiares precisam estar atentos a essas questões (CLAUMANN et al, 2018).

Além disso, a adolescência é um período de intensas modificações no desenvolvimento humano, marcado por alterações biológicas da puberdade e relacionado à maturidade biopsicossocial do indivíduo.

Desse modo, é identificada como um período de crise, pela experiência de importantes transformações mentais e orgânicas capazes de proporcionar manifestações peculiares em relação ao 
comportamento normal para a faixa etária (JATOBÀ; BASTOS, 2007).

Estas podem, contudo, ser confundidas com doenças mentais ou manifestações comportamentais inadequadas por isso a importância de se investir na formação de professores voltada para o tema do suicídio, de modo que o professor possa atuar de forma precoce na detecção desses casos (JATOBÀ; BASTOS, 2007).

Segundo Steinberg e Morris (2001), "nenhuma tentativa de desenvolver uma teoria geral do desenvolvimento adolescente normal encontrou ainda ampla aceitação", mas Haarasilta (2003) considera que as pesquisas sobre o desenvolvimento natural do adolescente são ainda escassas, o que torna plausível admitir a necessidade de mais bem conhecer as modificações psíquicas dessa fase da vida, por isso a necessidade de se desenvolver mais pesquisa e trazer o sistema educacional também par ao centro dessa discussão e não deixar somente para o setor da saúde.

\section{CONSIDERAÇÕES FINAIS}

O comportamento suicida em adolescentes vem sendo alvo de muitas pesquisas, porém relativamente poucas têm dado atenção específica aos fatores associados à etapa em que o sujeito não apenas pensa, mas já planeja como fará para acabar com a própria vida (BAGGIO et al., 2009).

Destaca-se a importância da efetiva capacitação dos professores a fim de que possam trabalhar temas importantes da vida desses jovens, identificarem adolescentes em risco e realizar ações que propiciem o maior envolvimento da família.

Além disso, os estudos apontam que o uso de drogas está diretamente associado ao pensamento e plano de suicídio e, entre as moças, o consumo de bebidas alcoólicas foi um fator associado ao estresse psicossocial.

Com isso, fica evidente que vários fatores colaboram pra o suicídio dos adolescentes, que é necessário que os professores estejam bem capacitados para a detecção precoce desses fatores, mas não só a vontade do suicídio, mas também os fatores concomitantes como o uso de drogas e bebidas alcoólicas tanto prevalente dentro da sociedade, e que a escola e sala de aula e o reflexo da sociedade.

Enfim, os achados enfatizam a importância dos fatores sinalizadores que podem permitir uma melhor compreensão sobre a problemática do suicídio e a participação da escola como um todo nesse processo.

\section{REFERÊNCIAS}

ARAUJO, Luciene da Costa; VIERA, Kay Francis; COUTINHO, Maria da Penha de Lima. Ideação suicida na adolescência: um enfoque psicossociológico no contexto do ensino médio, v. 15, n. 1, p. 47-57, jan./abr. 2010.

BAGGIO, Lissandra; PALAZZO, Lílian S.; AERTS, Denise Rangel Ganzo de Castro. Planejamento suicida entre adolescentes escolares: prevalência e fatores associados, v. 25, n. 1, p. 142-150, 2009.

BARROS, Airton Pereira Rêgo; COUTINHO, Maria Penha Lima; ARAÚJO, Ludgleydson Fernandes; CASTANHA, Alessandra Ramos. As representações sociais da depressão em adolescentes no contexto do ensino médio. Estudos de Psicologia, 23(1), 19-28.,2006.

BAHLS, Saint - Clair. Aspectos clínicos da depressão em crianças e adolescentes: clinical features. Jornal de Pediatria, 78, 5, 359-366, 2002.

BORGES, Vivian Roxo; WERLANG, Blanca Susana Guevara. Estudo de ideação suicida em adolescentes de 15 a 19 anos. Estudos de Psicologia, 11(3), 345-351.,2006.

CARVALHO, Priscila Diniz; BARROS, Mauro Virgilio Gomes; LIMA, Rodrigo Antunes; MELO, Edilanêa Nunes. Condutas de risco à saúde e indicadores de estresse psicossocial em adolescentes estudantes do Ensino Médio, v.27, n.11, p. 2095-2105, 2011

CASTRO, Marta de Lima; CUNHA, Sergio Souza; SOUZA, Delma Paula Oliveira. Comportamento de violência e fatores associados entre estudantes de Barra do Garças, MT, v.45, n.6, p. 1054-61, 2011.

CLAUMANN, Gaia Salvador; PINTO, André de Araújo; SILVA, Diego Augusto Santos; PELEGRINI, Andréia. Prevalência de pensamentos e comportamentos suicidas e associação com a insatisfação corporal em adolescentes. J Bras Psiquiatra., v.20, n.1, p.3-9,2018.

FERREIRA, Débora.; SANTOS, Antônio. Júnior.; RIBEIRO, Olívia.; FREITAS, Miguel.; CORREIA, João. Vieira.; RUBIN, Kenneth. Isolamento social e sentimento de solidão em jovens adolescentes. Análise Psicológica, Lisboa, v. 31, n. 2, 2013.

GOUVEIA, Nelson; CARLINI - COTRIM, Beatriz; GAZAL CARVALHO, Cynthia. Comportamentos de saúde entre jovens estudantes das redes públicas e privada da área metropolitana do Estado de São Paulo. Rev. Saúde Pública, v.34, n.6, p.636-45, 2000.

JATOBÁ, Joana D’arc Vila Nova; BASTOS, Othon. Depressão e ansiedade em adolescentes de escolas públicas e privadas. J Bras Psiquiatra, 56(3): 171-179, 2007

LEITE, Marinês Tambara; NARDINO, Janaine; HILDEBRANT, Leila Mariza; SANTOS, Adriane Marinês; MARTINS, Ricardo Vianna. Gestão do Cuidado na Estratégia Saúde da Família: 
Revisão Narrativa. Rev. Aten. Saúde, São Caetano do sul, v. 14, n. 49, p. 72-77, jul./set., 2016.

MACHADO, Jean Carlos; BOLDORI, Jilia Diane Martins; DALMOLIN, Marcelo Dalton; SOUZA, William Cordeiro; BAZZANELLA, Sandro Luiz; KNAESEL, Walter Marcos; GOMES, Luis Paulo. Incidência de Tuberculose nos Presídios Brasileiros: Revisão Sistemática. Rev. aten. Saúde, São Caetano do Sul, v. 14, n. 47, p. 84-88, jan./mar., 2016.

STEINBERG Laurence; MORRIS Amanda Sheffield. Adolescent development. Annu Rev Psychol, 52: 83-110, 2001.

SILVA, Viviane Franco; OLIVEIRA, Helenice Bosco; BOTEGA, Neury José; MARIN- LEÓN, Letícia; BARROS, Marilisa Berti Azevedo Barros; DALGALARRONDO, Paulo. Fatores associados à ideação suicida na comunidade: um estudo de caso-controle. Caderno de Saúde Pública, 22(9), 18351843.,2006.

VIEIRA, Kay Francis Leal. Depressão e suicídio: uma abordagem psicossociológica no contexto acadêmico. 2008. 159.Dissertação (Mestrado), Universidade Federal da Paraíba, Paraíba, 2008.

WERLANG, Blanca Susana Guevara; BORGES, Vivian Roxo; FENSTERSEIFER, L. Fatores de risco ou proteção para a presença de ideação suicida na adolescência. Revista Interamericana de Psicologia, 39(2), 259- 266.,2005. 